\title{
RAG1 et RAG2, deux gènes adjacents qui contrôlent l'expression de l'activité recombinase dans les prélymphocytes $B$ et $T$
}

La production de la diversité des anticorps est la conséquence de la recombinaison variable de plusieurs segments d'ADN codant, après réarrangement, pour les immunoglobulines. Un même phénomène explique la diversité des récepteurs pour l'antigène des lymphocytes $\mathrm{T}(\mathrm{m} / \mathrm{s}$, supplément au $n^{\circ} 1$, vol. 5, p. 5 et p. 8). La recombinaison, qui survient dans les lymphocytes en cours de différenciation, est catalysée par une "recombinase " qui reconnaît des signaux spécifiques aux bornes des segments devant subir le réarrangement.

L'équipe de D. Baltimore, du Whitehead Institute, MIT, Cambridge, MA, USA, avait, en 1989, cloné un gène qui était capable de faire apparaître une activité recombinase dans des fibroblastes qui en sont normalement dépourvus [1]. Pour ce faire, des cellules NIH 3T3 avaient été cotransfectées avec de l'ADN génomique total et un plasmide comportant des segments devant subir un réarrangement du type immunoglobuline et récepteur $\mathrm{T}$ pour conférer à la cellule une résistance à un agent cytotoxique, l'acide mycophénolique. Les cellules résistantes à ce produit étaient supposées contenir une activité recombinase catalysant le réarrangement productif du gène de résistance. Par des expériences de transfections successives (des cellules réarrangé étant transfectées par l'ADN des clones de cellules résistantes issus de la première expérience de transfection), un fragment d'ADN de $18 \mathrm{~kb}$ fut isolé qui semblait capable, après transfert dans des cellules fibroblastiques, de commander l'apparition d'une activité recombinase. Le gène RAG1 (recombination activating gene), contenu dans le clone, fut alors décrit [1]. Malheureusement, son efficacité semblait faible, car un ADNc RAG1 n'était pas plus actif sur l'induction de la résistance à l'acide mycophénolique que l'ADN génomique total. L'examen du clone génomique contenant RAG1 démontrait alors que ce gène était ici tronqué, ne possédant pas son promoteur et sa région toute 5' terminale. En réalité, le pouvoir "réarrangeant" de ce clone était dû au fait qu'il contenait un second gène situé à $8 \mathrm{~kb}$ du premier, appelé $R A G 2$. Le transfert conjoint de séquences actives codant pour RAG1 et pour RAG2 était au moins 1000 fois plus efficace pour induire une recombinaison de type immunoglobuline que celui d'un seul de ces gènes. $R A G 1$ et $R A G 2$ codent pour des protéines, respectivement de 55 et $58 \mathrm{kDa}$, qui ne sont pas homologues entre elles et ne ressemblent pas à d'autres protéines connues. Ces deux gènes ont la particularité d'être, apparemment, dépourvus d'intron. Les messagers RAG1 et RAG2 sont exprimés dans les cellules ayant une activité recombinase (prélymphocytes $\mathrm{B}$ et $\mathrm{T}$ ) et pourraient coder pour les deux sous-unités d'une même protéine multimérique. Formellement, cette protéine (ou ces protéines) pourrai(en)t être un(des) inducteur(s) de l'activité recombinase... ou constituer un(des) élément(s) essentiel(s) de la recombinase elle-même. L'absence d'homologie entre RAG1 et RAG2, d'une part, et des protéines de contrôle de l'expression de gènes, d'autre part, milite plutôt en faveur de la seconde hypothèse, de même que l'absence apparente d'activation d'autres gènes lymphocytaires dans les fibroblastes exprimant après transfection RAG1 et RAG2.

A. $\mathbf{K}$.

1. Schatz DG, Oettinger MA, Baltimore D. The $\mathrm{V}(\mathrm{D}) \mathrm{J}$ recombination activating gene, RAG1. Cell 1989 : 59 : 1035-48.

2. Oettinger MA, Schatz DG, Gorka C, Baltimore D. RAG1 and RAG2, adjacent genes that synergistically activate VDJ recombination. Science $1990 ; 248: 1517-3$. 